\title{
Compositional and thermal characteristics of palm olein-based diacylglycerol in blends with palm super olein
}

\begin{abstract}
Palm olein-based diacylglycerol (POL-DAG) was blended with palm super olein (POoo) in various concentrations (10-90\%), with increments of 10\% (wt/wt) POL-DAG. The physical and chemical characteristics, i.e., iodine value, acylglycerol content, fatty acid composition, melting and crystallization profiles and solid fat content, for POL-DAG, POoo and their binary blends were evaluated. The mid-infrared FTIR was used to determine the absorption bands of the different concentrations of the oil blends. Only slight differences of FAC and IV were observed. POL-DAG:POoo blends showed significant changes $(p<0.05)$ in DAG content and decreases in TAG content with increasing POL-DAG content. The DSC thermograms showed that the addition of different concentrations of POL-DAG changed the melting and crystallization behavior of the oil blends (POL-DAG:POoo). The crystallization onset point increased $(\mathrm{p}<0.05)$ with an increasing POL-DAG concentration $(10-90 \%)$. POLDAG has the same absorption bands as POoo, with the exception of several minor peaks that appeared at (I) $2954 \mathrm{~cm}^{-1}$, (II) $1267 \mathrm{~cm}^{-1}$, (III) $1199 \mathrm{~cm}^{-1}$, (IV) $1222 \mathrm{~cm}-1$ and (V) 966 $\mathrm{cm}-1$. This study will provide essential information for the palm oil industry to identify the most suitable POL-DAG blends with desirable physicochemical properties for food application purposes.
\end{abstract}

Keyword: Fat blends; Melting and crystallization; Palm olein-based diacylglycerol; Palm super olein; Acylglycerol 